\title{
Tracking of Fuel Road Tankers for Anti-Pilferage and Anti-Adulteration
}

\author{
Pallavi S. V \\ Department of ECE \\ Sambhram Institute of Technology, \\ Bangalore \\ Megha B \\ Department of ECE \\ Sambhram Institute of Technology \\ Bangalore
}

\author{
Aakanksha Dubey \\ Department of ECE
}

Sambhram Institute of Technology, Bangalore

\author{
Piya P. Saha \\ Department of ECE \\ Sambhram Institute of Technology \\ Bangalore
}

\author{
Ezhilarasan $\mathrm{K}$ \\ Department of ECE \\ Sambhram Institute of Technology \\ Bangalore
}

\begin{abstract}
Fuel adulteration and pilferage are becoming a global issue nowadays. Transportation facilities must be better enough for the overall development of the country, which basically depends on the quality and purity of the fuel used in automobiles/vehicles. The term "Adulteration" basically refers to the process of adding few adulterants to degrade the quality of the fuel and also to increase the density of the fuel quantity. to altering the properties of fuel, it doesn't meet the specifications as per requirement. Use of adulterated fuel in automobiles causes the exhaust gases to be emitted from vehicles which is a major source of urban air pollution in this modern world. Adulteration of gasoline and diesel further provokes the problem. In order to overcome the adulteration, monitoring of fuel quality at the loading and unloading end, therefore, is essential. The term "Pilferage" basically refers to stealing or thefting. Oil theft always results in huge economic loss, human causalities, and extremely environment pollution especially when the leaks from crude oil pipeline are not detected and repaired timely. The intelligent anti-theft system application can not only stop oil theft timely, but also prevent oil mice from stealing crude oil before-hand. An efficient automotive security system is implemented for anti-theft detection using embedded system consists of Global Positioning System (GPS) and a WI-FI module. The system in the event of theft will send a predefined message to the owner of vehicle. The aim of this project is firstly to monitor for fuel leakage to avoid fire accidents providing safety feature where security has been an important issue. Secondly an alert message will be sent when the density of the fuel changes due to the pressure or thefting. Third is an alert signal or message is sent when the fuel tanker doesn't reach the destination and its exact location and activities are monitored in real time and lastly it checks the quality of fuel based on parameters (like density, temperature, viscosity etc.)
\end{abstract}

\section{Keywords}

Pilferage, Adulteration, Degradation, Real time monitoring, Parameters, Fuel tankers etc

\section{INTRODUCTION}

Crude oil stealing always results in huge economic loss, environment pollution and human causalities in the world. In China, oil companies lost about more than three billion Yuan every year. Then, the oil thieves named oil burglars who know oil companies' operations of detecting technology well began to rob crude oil by pumping from oil tanks or drilling a hole into oil pipeline. The oil burglars are becoming millionaires at one night even relatively small amounts when oil price is very high, and oil companies suffer a huge economic loss at the same time. These oil companies undergo extreme economic loss and negative social influences on society so that most of them are determined to set up perfect intelligent monitoring system. This magnitude system might cost millions of dollars or even more, which is not a big issue when compared to billions of economic losses that the oil theft result in. So how to make use of advanced information technology to improve the current techniques that only detect change of pressure in oil tanks is the problem to be addressed urgently all over the world.

Petroleum is one of the important transportation fuels. It occurs naturally below the earth surface. It is processed and converted into various types of fuels called gasoline. Adulterants alter the chemistry of base fuel so the product will not meet the requirement and specification which interns affect the internal combustion engines giving harmful pollutants in the atmosphere. Fuel adulteration are rampant globally and is surreptitious operation in which higher priced fuel is mixed with cheaply available substitute. The adulteration intern damages the engines of the vehicle leading to the emission of harmful gases causing pollution and affecting the human survival.

\section{RELATED WORK}

Jinfeng Sun et.al [1] proposed the intelligent crude oil antitheft system based on IOT under different scenario oil theft always results in huge economic loss, human casualties, and extremely environmental pollution especially when the leaks from crude oil pipeline are not detected and repaired timely. In this paper, we focus on how to detect and monitor abnormal noise and vibration beforehand or in real time by the Internet of Things (IOT). Firstly, the diversities of crude oil theft and the difficulties of oil anti-theft are analyzed in China, and the requirement analysis of the IOT application is stated. Secondly, the intelligent antitheft system based on the IOT is planned and designed for crude oil transportation by tank trucks and by oil pipelines according to the current situation in China. Thirdly, the problems of anti-theft system implementation are discussed, and the suggestions and advices are put forward to ensure that the system can be implemented successfully. The intelligent anti-theft system application cannot only stop oil theft timely, but also prevent oil mice from stealing crude oil beforehand. The drawback of 
this paper is Quality of fuels is not checked which is also important factor in terms of economics.

R.K. Sharma, Anil Kumar Gupta [2] proposed Detection/ Estimation of Adulteration in Gasoline and Diesel using Ultra sonics. The exhaust gases from fast increasing number of automobiles is a major source of urban air pollution in third world countries. Adulteration of gasoline and diesel further aggravates the problem. For the prevention of adulteration, monitoring of fuel quality at the distribution point, therefore, is essential. In this paper the feasibility of detection/estimation of adulteration in gasoline and diesel using ultrasonic radiations has been proposed. The speed of ultrasound in nonadulterated and intentionally adulterated gasoline and diesel has been determined using pulse echo method. It has been observed that adulteration results in the change in the measured speed of ultra sound which can be calibrated in terms of percentage adulteration.

It is not efficient method for large quantity of fuel because it cannot measure the amount of impurity added for large quantity of fuel.

Pranjali P. Dharurkar, Arti R. Wadhekar [3] Proposed Estimation of Petrol Adulteration using Statistical Feature Analysis Approach. Fuel adulteration has become a very important and global issues nowadays. For overall development of the country transportation facilities must be better which depends on quality and purity of fuel. Adulteration or mixing of various substances causing degradation and reducing the quality of fuel has become an important issue nowadays, due to which overall economy of the country is down. Due to altering the properties of fuel, it doesn't meet the specifications as per requirement. The proposed system is based on Statistical analysis of the data samples. The system is based on Image analysis by which various parameters are calculated. The Existing systems are quite tedious and time consuming. Kerosene and diesel are the adulterants used in petrol. Fuel-adulterant mixtures in different proportions by volume were prepared and individually tested. The mixtures were administered to vehicles and the tail pipe exhaust emission drastically increased. There is considerable change in (Peak Signal to Noise Ratio), and Histogram plot shows the differences of Standard and adulterated concentrations. The proposed system results are validated against Laboratory Tests. The drawback of this paper is Lack of ability to implement in real time application successfully.

Areeg Abubakr Ibrahim Ahmed et.al [4] discuss Fuel Management System. Monitoring Systems are necessary to track and understand the changes that take place in environments. Remote monitoring and data collection systems are useful and effective tools to collect information from fuel storage tanks. The fuel management system is a monitoring device built on the Raspberry-Pi computer, it takes information about tank's fuel level in real time through its sensor and live streaming of the site, then uploads it directly to the internet, where it can be read anytime and anywhere through web application. This paper presents the implementation of monitoring system based on internet of things technology to protect the tower sites from theft and provide security to remote locations. The drawback of this paper is that, in recent years, Fuel use safety cannot be optimistic. Currently, the main Fuel network detection is the use of portable / handheld Fuel leak detectors, vehicle mounted Fuel network monitoring system, and the disadvantage is that the both detection systems cannot achieve real time Fuel information collected. So, we go for the sensor based proposed system to achieve and overcome the disadvantage in the earlier system.

Sarvraj Singh Ranhotra [5] provides the survey of Checking automobile fuel adulteration using Image Processing Techniques. Different samples of automobile fuel, petrol contaminated with different proportions of kerosene, and thereby proposing a sensor for checking adulteration of fuel using image processing techniques. Images of different samples are captured using a camera and image processing is employed to extract the trends of measures of texture analysis. The findings show that adulterating fuel with different proportions of impurities display varying characteristics of five measures of texture. This research is enormously significant to the development of sensors to detect fuel adulteration. Since the inception of the automobile industrialization, air pollution has augmented due to consumption of fossil fuels. Automobile industry being the major consumer of fuel oil, are the main culprits for the rising levels of air pollution. Adulteration of automobile fuels leads to increased exhaust pipe emission and the detrimental impacts in the atmosphere. The adulteration of petrol using kerosene is difficult to detect and is financially alluring. The expected adulteration percentage is $15 \%$ to $35 \%$ by volume. Any figure beyond this range can be detected by the automobile user by the degrading engine performance. To counteract adulteration effectively, it is necessary to determine the fuel quality at the distribution source. The devices implemented should be portable and computationally fast, capable to provide the results quickly, should be preferably inexpensive for easy deployment of such equipment's. Several methods have been proposed by The American Society for Testing and Materials International. To list a few, Evaporation Test, Distillation Test, Gas Chromatography and Adulteration Estimation/Detection using Optical Fiber Sensor. Above mentioned techniques are only viable if provided with sophisticated instruments. Here a method using human perception and image processing techniques has been proposed to check fuel oil adulteration. In the past, optical sensors have been used to check adulteration. The use of Fiber Bragg gratings to distinguish amongst various samples of adulterated petrol. The next improvement was the use of etched Fiber Bragg gratings which are used as a chemical sensor for checking adulteration. Also, the speed of ultrasonic have been found to be varying when passed through different mediums, therefore passing through a sample of adulterated petrol would confer different observations.

Capturing the image top view of the samples of petrol with various proportions of kerosene as impurity, the procedure follows background subtraction technique. Here rudimentary morphological processes and texture analysis are applied to detect the idiosyncratic characteristics of the samples. After capturing images, image enhancement techniques, segmentation and morphological operations were applied followed by the texture analysis.

Sanjay Kher, Smita Chaubey, Jai Kishor and Shrikant M. Oak et.al [6] proposed Detection of Fuel Adulteration with High Sensitivity Using Turnaround Point Long Period Fiber Gratings in B/Ge Doped Fibers, Adulteration of automobile fuel i.e. kerosene in petrol, leads to air pollution and the consequent ill effects on public health. The automobile fuel industry is an area which is in constant search of sensors for in-situ monitoring of quality control and adulteration detection. The adulteration detection of kerosene in petrol is a vital problem since there is no standard universal technique 
for quantitative measurement of adulteration. Since kerosene is cheaper than petrol, kerosene is usually added to petrol for monetary gains. Adulteration of kerosene in petrol changes the refractive index of petroleum and therefore, optical fiberbased sensors capable of measuring the refractive index changes can be used for detection of adulteration. These sensors offer unique characteristics such as ease of fabrication, high sensitivity, fast response, compact footprint, resistance to harsh environments besides being chemically inert and immune to electromagnetic interference.

Rajneesh K. Verma, Payal Suwalka et.al [7] proposed Detection of adulteration in diesel and petrol by kerosene using SPR based fiber optic technique This paper was focused on the experimental investigations for fabricating a surface plasmon resonance (SPR)based fiber optic sensor for the detection of the extent of adulteration in petrochemicals: petrol and diesel by kerosene. Primarily it is observed that the refractive index of the petrol and diesel changes if we mix kerosene in it. The variation in refractive index is linear in nature. Utilizing the phenomenon of surface plasmon resonance in Krestchmann configuration on optical fiber, the percentage of adulteration in petrol and diesel is detected. The detection level of adulteration is quantified systematically for both the petrol and diesel. The study carried out here explores the possibility of utilizing SPR technique for the detection of the level of adulteration in petrochemicals. The suitability of the optical fiber for remote sensing and its immunity towards electromagnetic interaction makes this probe very useful for such endeavor. High sensitivity, easy construction and its portability, makes this study important in the development of SPR based optical fiber sensors for petrochemical industries. Apart from these various aspects of environment polluting hazardous/toxic gases as an emission product of automobile fuels has also been discussed.

Vimal Babu, Rama Krishna, Naga Mani [8] provided the Review on the Detection of Adulteration in Fuels through Computational Techniques. The scope of the paper is to review different works on fuel adulteration process detection using computational techniques. Gasoline (Motor Spirit/MS) and Diesel (High Speed Diesel/HSD) are major fuels used for transport/Electricity generation universally. Consumers are facing the threat of fuel adulteration. Adulterants are added to these fuels with other cheaper boiling point range hydrocarbons having more or less similar composition leading to alter and degrade the quality of the base fuels. These adulterants are added by the business community for their monetary gains. Detecting such adulterant in fuels is a foremost task in the interest of the end consumer. The petroleum products are being utilized in transport, power generation as fuel, universally in day to day life. The consumption is increasing every year to the tune of $4 \%$. The businessmen who sell the transport fuels are adopting dishonest techniques for their profit by adding low cost hydrocarbon additives to the base fuel. This is causing environmental pollution. The pollutants coming out as tailpipe exhaust is posing a great threat both to the environment as well as human health. To check, control the adulterants both in Diesel and Gasoline there shall be perfect mechanism both at laboratory level as well as at statutory level. One such method to analyze the fuel adulteration is Artificial Neural Networks which is one of the accurate methods than any other existing methods.

Danyelle A. Cunha, Luciana F. Montes et.al [9] proposed a technology NMR in the time domain. A new methodology to detect adulteration of diesel oil with kerosene. Petroleum products such as LPG (cooking gas), kerosene, gasoline, diesel oil, among others, are obtained 24 from the refining of crude oil. Among these, the diesel has great importance in the Brazilian road system 25 where fuel consumption leads the rankings in the road sector in Brazil. The high consumption (approximately $50 \%$ of total) makes this fuel target to adulteration by irregular products like kerosene, which has low cost and good miscibility with the fuel. Adulteration causes irreparable damage to the car, such as sudden drops in engine, difficulties in starting, increased fuel consumption, degradation of machine performance and environmental harm as emissions of particulate matter during the combustion. This study proposes a new methodology, the nuclear magnetic resonance in the time domain (TD-NMR) to detect the adulteration of four commercial diesel oils with kerosene. Results of the study show that the diesel oil has a transverse relaxation time (T2) around $0.67 \mathrm{~s}$, while the adulterant kerosene is $1.33 \mathrm{~s}$, and the 33adulterated samples in the range of $5-100 \%$ presented $\mathrm{T} 2$ between 0.67 and $1.33 \mathrm{~s}$. The TDNMR methodology proposed showed excellent agreement with quantification of adulterant content detected by midinfrared (MIR) which is the standard technique to detect noncompliance of fuel.

Vinoth Kumar, Upendran Rajendran et.al [10] proposed Antitheft control system design using embedded system. The antitheft control system for automobiles tries to prevent theft of vehicle. This system makes use of an embedded chip that has an inductive proximity sensor, which senses the key during insertion and sends a text message to the owner mobiles stating that car is being accessed. This is followed by the system present in the car asking the user to enter the unique password. The password consists of few characters and the key number. If the user fails to enter the correct password in three trials, a text message is sent to the police with the vehicle number and the location tracked using a GPS module. The message is also sent to owner about the unauthorized usage. Further the fuel injector of the car is deactivated so that the user cannot start the car by any means. At the same time a secret lock system activated and unauthorized user get trapped inside the car and only the owner wo is equipped with the key to the secret lock system can deactivate the mechanism. This technique helps in taking the fast steps towards an attempt to steal. The design is robust and simple.

Daniel.B.R. Rodrigues, Henrique E.M. Peres, Wesley Becari et.al [11] proposed Ethanol Fuel analysis by Time domain Reflectometry, several samples of alcohol with the addition of its main contaminants (water and methanol) were prepared and measured with the TDR sensor. The results indicate good response linearity, showing the TDR technique is a promising technique for fuel qualification. There are numerous problems caused by illegal adulteration of automotive fuel commercialized in Brazil. First, adulterated fuel results in reduced engine lifetime. In extreme cases, the so called "engine knocking" or "detonation" can occur, when the combustion process loses its synchrony. This effect can make some of the engine parts (such as the piston) burn completely, rendering them useless. Another problem involves the pollution resulting from "bad" fuel burning. The production of toxic gases is unavoidable in fuel combustion, but the use of adulterated products results in far more dangerous pollution. Methanol, used to adulterate ethanol, can cause permanent blindness or even death in sufficiently large doses. The country national economy also suffers due to adulterated fuels. One of the main reasons why occurs is the different amount of tax revenue (illegal compounds are cheaper). Tax evasion results in the creation of unfair competition and 
irregular tax payment. Usual fuel analyses often involve the use of large and expensive equipment and require destructive chemical methods. This work introduces studies of the TDR (Time Domain Reflectometry) technique as an auxiliary to the analysis, proposing a fast, simple, compact and nondestructive method for alcohol qualification. Being a widely known method in telecommunications, with "cable-tester", and in geotechnical studies, the relation between the TDR technique and the electrical permittivity of the medium under analysis might indicate it for fuel qualification.

Boris Levitas [12] proposed UWB based oil quality detection, an approach for fuel quality detection using Ultra-wideband (UWB) radar. The experimental setup consists of an ultrashort monocycle pulse transmitter (30ps), an UWB sampling receiver and UWB antennae (3.1-10.6 GHz). One dimensional Finite Difference Time Domain (FDTD) model was used to model the electromagnetic wave propagation and assess the feasibility of oil quality detection using UWB RADAR. Performance of the UWB RADAR was analysed through detailed experiments. The adulteration of gasoline and highspeed diesel with kerosene was increased from $1 \%$ to $12 \%$ in steps. Using time of flight analysis, the variation in adulteration of gasoline was detected from $1 \%$. Adulteration in high speed diesel was detected using frequency domain analysis. Further, the level of water in the oil storage tank below the diesel or gasoline level can be accurately estimated. Detailed experiments with multiple batches of gasoline and high-speed diesel were conducted. We also demonstrate that the adulteration can be detected across different batches of fuel. Fuel adulteration in the supply chain is a widespread issue in several countries around the world. Adulteration of automobile fuels such as gasoline and diesel with kerosene is very common. The main reasons for adulteration are: huge price difference between gasoline, diesel and kerosene, financial incentives arising out of differential taxes and easy availability of kerosene. Kerosene is an important domestic fuel used by mostly people below the poverty line and is heavily subsidized. Adulteration not only degrades fuel quality but also has adverse effects on the environment, public health and vehicle engines. Gasoline adulterated with high a percentage of kerosene displays octane quality far below the octane requirement of the engine and engine knocking can occur. The falling octane quality reduces the efficiency and performance of the engine and can also damage the engine mechanically. Fuel quality monitoring at the distribution centre or the retail outlet is essential to check adulteration and to reduce air pollution. A number of test methods based on analytical and field techniques have been developed to detect and estimate adulteration levels in gasoline and diesel. Most commonly used analytical test methods are evaporation technique (ASTD D 381), distillation technique (ASTD D 86), gas chromatography, chemical markers etc. A density test using portable hydrometer is most commonly used field technique. Field tests can produce results quickly but they are not as detailed as analytical methods. A number of other methods have been proposed in the literature to estimate fuel adulteration based on statistical analysis, ultrasonic, optical fibre, kinematic viscosity and microwave methods. The sensing principle in ultrasonic techniques is based on the change in the speed of ultrasound between non-adulterated and adulterated oil mixtures with increasing percentage of the adulterant. The reported results based on ultrasonic method use high levels of kerosene adulteration and calibration to estimate adulteration level. The fibre optic method is based on modulation of the intensity of light guided in the fibre due to change in the refractive index of cladding formed by adulterated fuel. Solutions based on the fibre optic method are expensive and contact based as the fibre is immersed in the fuel. Microwave systems employing FSCW (Frequency Step Continuous Wave) radar systems transmit a sequence of sinusoids at different frequencies and measure the steady-state amplitude and phase shift induced by the channel to estimate impurity levels such as the sludge at the bottom of storage tanks. Error of few percent in determining the dielectric constant can lead to a high error in estimating the impurity level in the tank. The reported error in the estimate of dielectric constant using FSCW radar is about 1\%. In this paper, we propose a simple, non-contact-based fuel quality monitoring of gasoline and high-speed diesel adulterated with kerosene using the UWB technology. UWB based solutions are cost effective and very attractive due to low-power requirement, simple transceiver model, high processing gain, high throughput, co-existence with other wireless technologies and low multipath fading effects. UWB RADAR operates by radiating electromagnetic waves with pulse widths of the order of sub-Nano seconds into oil samples and analysing the reflected signal for information about the dielectric constant and other parameters of the oil samples.

R. Yadav, Murthy V, Mishra et.al [13] provided the Estimation of Petrol and Diesel Adulteration with Kerosene and assessment of usefulness of selected automobile fuel quality test parameters. The common adulterants used in mixing of diesel is Kerosene. Five fuel adulterants with different proportion of volume were prepared and tested individually for density and kinematic velocity. These mixtures were tested with the vehicles and the exhaust emission test was done for opacity value. There was no variation at the different levels of adulterants. Even at higher Adulteration the density was within the prescribed value. At higher Adulteration levels there was a change in kinematic velocity. Even at small level of adulteration the percent opacity value was decreased sharply. The amount of Kerosene present in diesel as $35 \%$ to $50 \%$ on the filling stations of Kathmandu city. This suggested that the density test was not the accurate form of checking the adulteration. The useful Adulteration parameters are kinematic velocity and opacity values. The Adulteration process is a covert and profit bearing operation. The common practice now a days is mixing up of Kerosene with diesel. Many vehicles run on Adulterated fuel. The objectives of the study were analyzing the usefulness of density and kinematic velocity tests applied to different proportional mixtures of fuel and Adulterants and also the analyze the variations in the opposite value of the Adulterated fuel. Materials and methods procurement of pure diesel: From army petrol filling station the diesel of about 32 liters was obtained. The purity of diesel was estimated by checking the density and kinematic viscosity and by comparing these values with the standard values. the kerosene of about 26lts was added to it from a local petrol pump. Preparation of custom proportions mixture of fuel and adulterant: Diesel and kerosene were mixed volume by volume in six different proportions. The volume which was prepared finally was 81ts and this mixture was sent for testing emission separately for the opacity value.

Laboratory tests:

The tests conducted in laboratory were density and kinematic viscosity tests. For density estimation calibrated hydrometers and thermometers are used.

Amit P. Gawande et.al [14] proposed Fuel adulteration consequences in India. The rate of petroleum utilization in our country is raising due to urbanization, development activities, 
population which leads to the widespread population in environment. As there is an increase in the fuel price the tanker drivers blend the petroleum products. The blending may be from $80-90 \%$. One of the major consequences of fuel adulteration is air pollution, which is not a unique problem in India. In this paper development techniques for fuel adulteration with kerosene which causes environmental impacts has been described. Different products of different quality have variable prices where the consumers do not have

efficient tool to distinguish same products of different qualities, therefore unethical operators will try to exploit the situation for illegal benefits. Mal practices in the retail business are one of the global phenomena, and fuel adulteration is one of the major practices. These leads to losses in several areas such as damaging the vehicles engines and polluting the air. Adulterating gasoline with kerosene can make a harmful deposit in the engine. In India the adulteration of gasoline mainly takes place due to the difference in the price of the products. Adulteration refers to the addition of another substance to the fuel in order to increase the quantity of the fuel, which may result in the loss of actual quality of the fuel. Here the foreign substances are called as adulterants which alters and degrades the quality of the transport fuels. Adulteration takes place during the process of transporting the fuel and during selling it. The transport fuels have been adulterated with other bi-products for the monitory gains. For example, gasoline is been adulterated with kerosene. Since the adulterants are easily available in the market, the adulteration rate has been increased drastically in the past few years. The primary cause of fuel adulteration is financial incentives which arises from differential taxes. Since there is a significant price difference between products and the adulterant they are indulged in adulteration.

Some of the effects of adulteration are:

1. Failure of components, no proper functioning of the engine.

2. Increases the emission of hydrocarbons, oxides of nitrogen, carbon monoxide, these causes the increased emission.

3. These causes health problems. It may be noted that all forms of adulterants are not harmful to public health.

4. Significant loss of tax revenue: This results to the financial losses of the country.

Types of fuel adulteration:

1. Blending of lubricants into kerosene as a substitute for diesel.

2. Blending of kerosene into diesel.

3. Blending of kerosene into petrol.

4. Blending of lubricants into diesel.

Adulterants exists in various forms and it varies from place to place depending on the place and quality. The factors which govern the choice of adulterants are blend ability, availability and profitability. Fuel adulteration reduces the quality of the fuel which in turn effects the combustion dynamics inside the combustion chamber which releases harmful pollutants. The consequences such as air pollution, quality of life and effect on health, reduced life span of vehicle engine is been ignored now-a-days.
The parameters which are required for the testing of fuel are:
1. Density
2. Distillation
3. Stability
4. Octane number
5. Multifunctional additives-dosage.
6. Hydrocarbon consumption

Matthias W eifi, R Einhard et.al [15] proposed Novel methods of measuring impurity levels in liquid tanks. To find out the level of the liquid surface in storage tankers conventional FMCW radar technique is implemented. In the second case it also detects the impurities such as water in the petrol tank. The dielectric constant of the petrol should be known to detect the impurity level in the tanker. Uncertainties of $0.2 \mathrm{~mm}$ for different levels with frequencies from 1.5 to $3.5 \mathrm{Ghz}$. The important industrial purpose is to measure and control the liquid levels in the tank. The impurities of the contaminants may settle in the storage tanks in the mineral oil industries. monitoring of this is required so that the impurities do not reach the next level. the storage tanks had a scheduled maintenance, this scheduled maintenance could be replaced by on - call maintenance if the contaminant level is known. The dielectric constant of the fuel is needed to calculate the physical liquid levels. The advantage of this algorithm is foe determining the dielectric. this finds its application in industries where the impurity level is to be calculated. For example, in the Petro-chemical industries water is always present. By having an accurate value of the impurity level regular scheduled maintenance can be replaced by maintenance as required. 


\section{SYSTEM DEVELOPMENT}

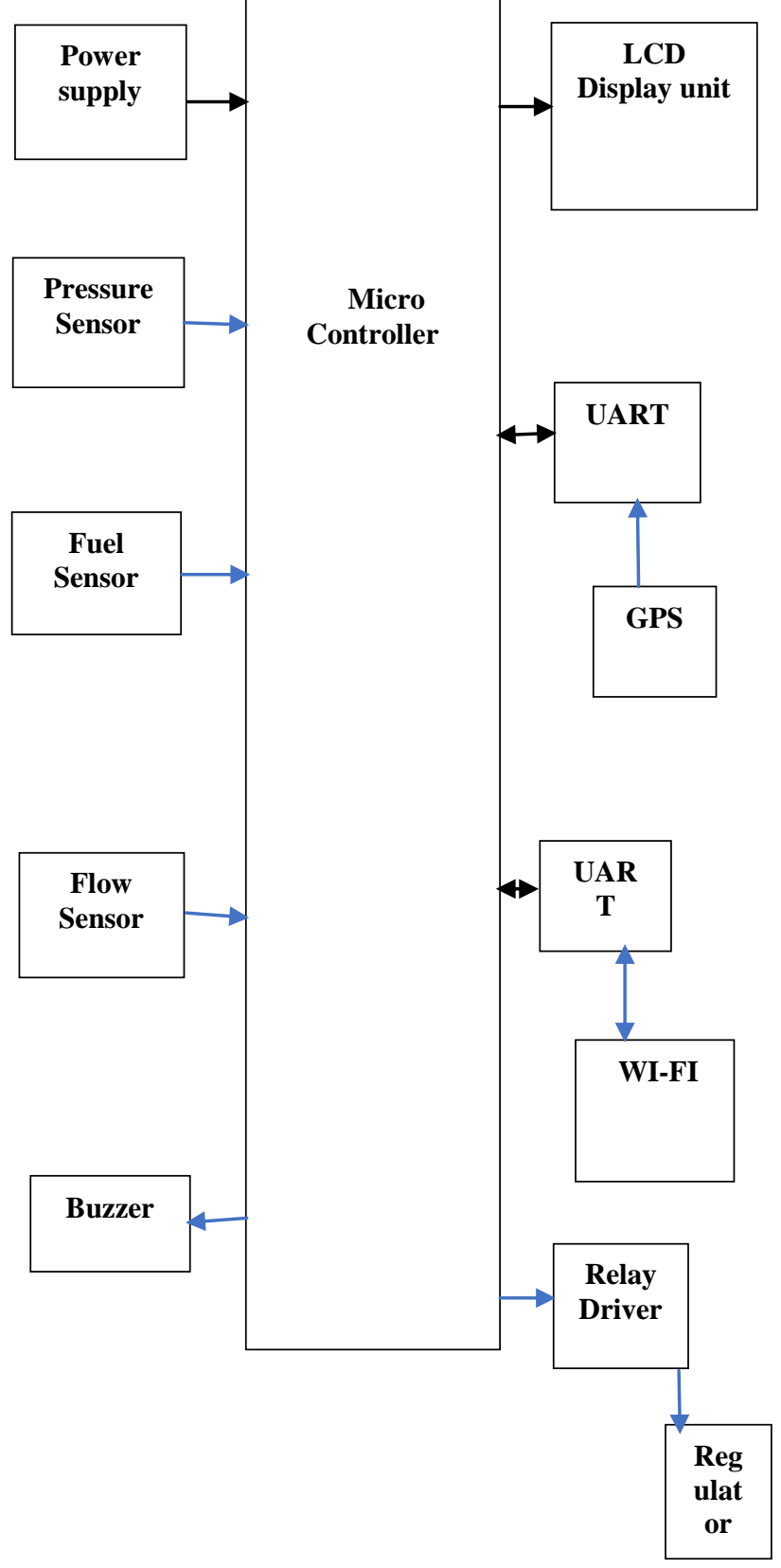

Fig. (1) Block Diagram of proposed system.

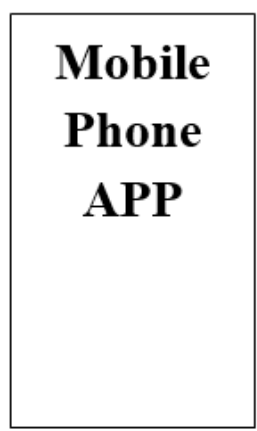

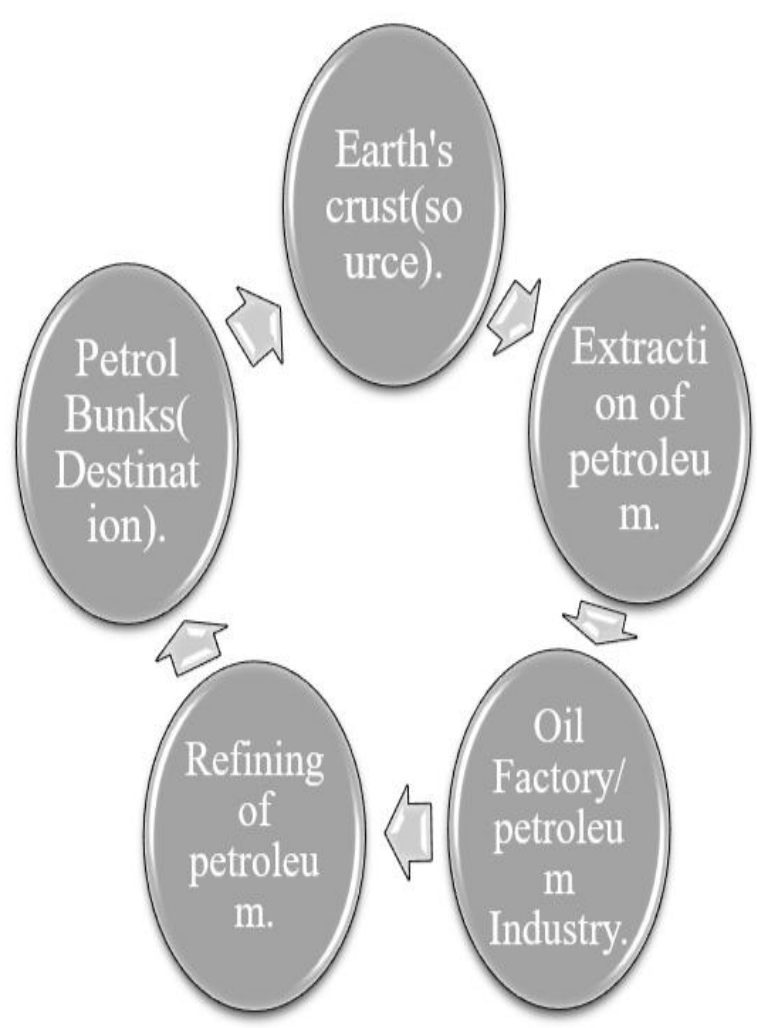

Fig. (3) Petroleum extraction process.

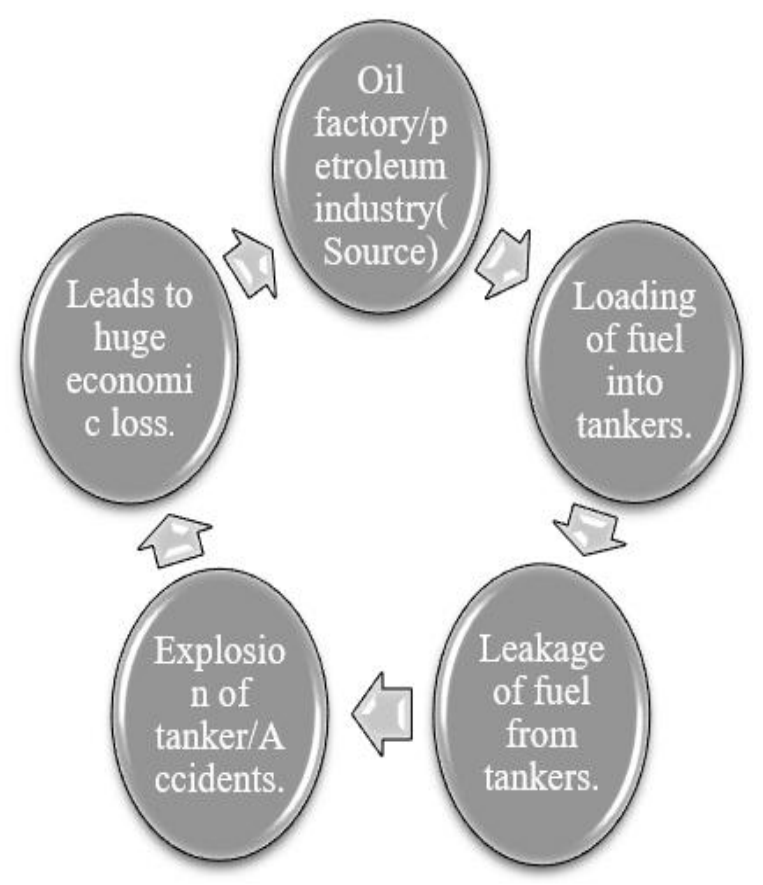

Fig. (4a) Fuel Thefting (Pilferage) Process.

Fig. (2) Collector Section. 


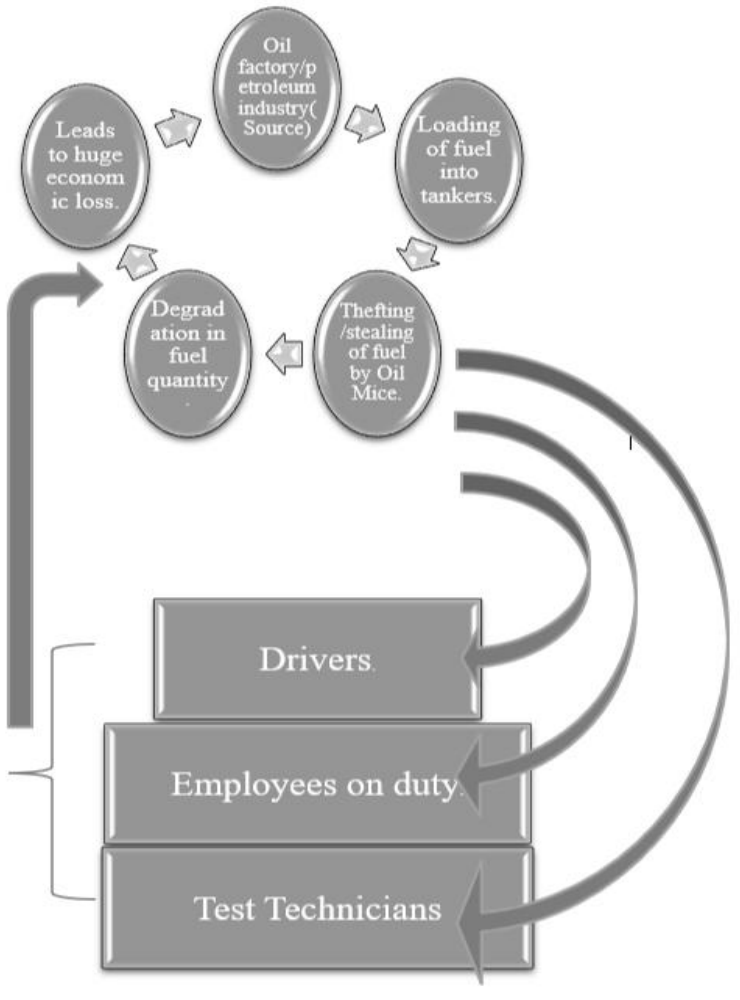

Fig. (4b) Fuel Thefting process.

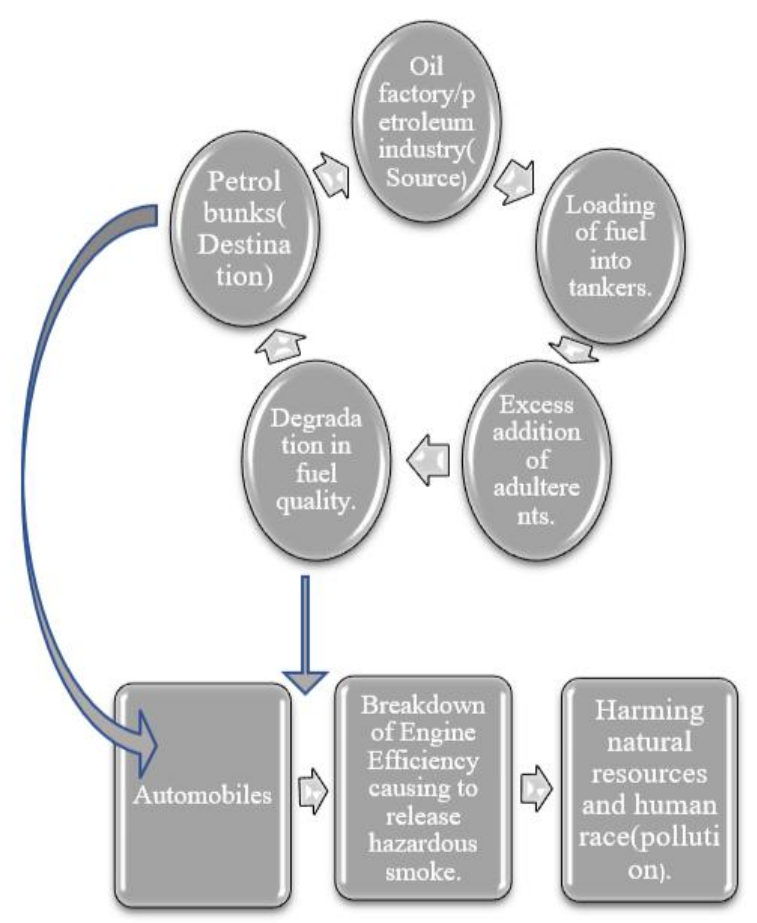

Fig. (4c) Fuel Adulteration Process.

The proposed system basically consists of pressure sensor which is usually used to detect the variation in pressure level within the tanker (closed loop) due to long distance travel. Increase in pressure in-turn increases temperature leading to a spark inside the tanker causing blast or explosion of vehicle affecting the economic, natural and human resources.

Fuel sensor plays a major role in detecting the leakage from tanker through valves or other means. Flow sensor is used to detect the variation in the level of fuel. Through GPS mechanism the real time activities, location and its status are monitored. Finally, Buzzer acts as a means of alarm for the driver in any of the above-mentioned critical situation so that the proper measures could be taken in order to come it without causing any harm. And also, other sensors are used to measure the quality or purity of fuel based on parameters (temperature, density, viscosity, pressure, color etc.)

\section{CONCLUSION}

An overall view can be concluded as, the device designed for tracking fuel road tankers for Anti-pilferage and AntiAdulteration is technically feasible to the petroleum industry domain and Government. It is based on ARM microcontroller provided GSM service using trending technology that is IOT. It gives real time information always, even in rural areas which in-turn helps to take the remedial measures in time to avoid the occurrence of disasters/destructions for nature and human resources. It is very helpful. It gives ease of access also. By implementing these ideas, the percentage for appropriate fuel pilferage and fuel adulteration information retrieved can be improved.

\section{REFERENCES}

1. Jinfeng Sun, "The Intelligent Crude Oil Anti-Theft System Based on IoT Under different scenerios" https://doi.org/10.1016/j.procs.2016.08.205

2. R. K. Sharma, Anil Kumar Gupta, "Detection/ Estimation of adulteration in gasoline and diesel using ultra

https://doi.org/10.1109/ICIINFS.2007.4579230

3. Pranjali P. Dharurkar and Arti R. adhekar, "Estimation of Petrol Adulteration using Statistical Feature Analysis Approach" https://ieeexplor.ieee.org/document/7530329

4. Areeg Abubakr Ibrahim Ahmed, "Fuel management system" http://doi.org/10.1109/ICCCCEE.2017.7867671

5. Sarvraj Singh Ranhotra, "Checking automobile fuel adulteration using Image Processing Techniques" https://ieeexplore.ieee.org/document/6707661.

6. Sanjay Kher, Smita Chaubey, Jai Kishor and Shrikant M. Oak, "Detection of Fuel Adulteration with High Sensitivity Using Turnaround Point Long Period Fiber Gratings in $\mathrm{B} / \mathrm{Ge}$ Doped Fibers" https://ieeexplor.ieee.org/document/2270312

7. Rajneesh K verma, Payal Suwalka, Jatin Yadav, "Detection of adulteration in diesel and petrol by kerosene using SPR based fiber optic technique" https://doi.org/10.1016/j.yofte.2018.04.011

8. Vimal Babu, Rama Krishnan, naga Manic, "Review on the detection of adulteration in fuels through computational techniques" https://doi.org/10.1016/j.matpr.2017.02.013

9. Danyelle A cunha, Luciana F. Montes, Eustaquio V. RvCastro, Lucio L. Barbara, "NMR in the time domain: A new methodology to detect adulteration of diesel oil with kerosene" https://doi.org/10.1016/j.fuel.2015.10.078

10. Vinoth Kumar Dagupan, Upendran Rajendran. Albert Joe Francis, "Anti-Theft Control System Design Using 
Embedded https://doi.org/10.1109/ICVES.2011.5983776

System"

11. Daniel B.R. Rodrigues, Henrique E. M. Peres, Wesley Becari, "Ethanol Fuel analysis by Time domain Reflectometry" https://ieeexplor.ieee.org/document/6646441

12. Boris levitas, Jonas Matuzas, Ganapathy Viswanath, Vijaykumar Basalingappa and Vijendra, "UWB based oil quality detection" https://ieeexplor.ieee.org/document/6058832
13. Sh. R. Yadav, V. K. Murthy, D. Mishra B. Baral, "Estimation of petrol and diesel adulteration with kerosene and assessment of usefulness of selected automobile fuel quality test parameters" https://link.springer.com/article/10.1007/BF03325839

14. Amit. P. Gawande, "Fuel Adulteration Consequences in India" https://www.tsijournals.com/articles/fueladulteration-consequences-in-india--a-review.pdf

15. M. Weiss and R. Knochel, "Novel methods of Measuring Impurity Levels in Liquid Tanks https://ieeexplore.ieee.org/document/596709 